Instability, Chaos and Predictability in Celestial Mechanics and

Stellar Dynamics
Copyright 1992 by Nova Science Publishers, Inc. All rights of reproduction in any form reserved. ISBN 1-56072-054-9

\title{
ChaOs AND ORder in THE Rotational MOtion
}

\author{
Andrzej J. Maciejewski \\ Institute of Astronomy, Nicolaus Copernicus University, \\ 87-100 Torun, ul. Chopina 12/18, Poland
}

\section{ABSTRACT}

It was proved that the problem of perturbed planar oscillations of a rigid-body in a circular or bit is nonitegrable. Two types of perturbations were considered: solar radiations pressure and the third body torques. In the second part of the paper example of chaotic rotations of a symmetric rigid body in a circular orbit was given. It was shown numerically that the phase space is divided into two separate regions of chaotic and ordered motions.

\section{INTRODUCTION}

There are many examples of simple dynamical systems with the so called chaotic behaviour. The main problems connected with such a behaviour seem to be still open. Will not discuss all possible mechanisms that lead to complex structure of phase curves and cause nonitegrability. The method of the separatrices splitting [8] was chosen as a tool for proving nonitegrability. In the cases considered here this method is effective and allows to obtain some information about 'nature' of chaos of the dynamical system.

Poincare [12] was the first who discovered that transversal crossing of asymptotic surfaces of unstable periodic solution leads to complex structure of phase curves. Melnikov's result [11] allowed to formulate theorems about nonitegrability of systems with transversal homoclinic (heteroclinic) orbits (see [19],[20],[3]). Below, we shortly describe basic 
points of the method employed that will be uses 1ater on. Proofs and details can be found in the cited references.

Let us consider $2 \pi-p e r i o d i c$ hamiltonian system with one degree of freedom. Hamiltonian function is assumed to ba analytic with respect to its arguments and depends on small parameter:

$$
H=H(x, t, \epsilon)=H_{0}(x)+\epsilon H_{1}(x, t)+\ldots, x=(q, p)
$$

We assume that unperturbed system ( $\epsilon=0)$ possess hyperbolic equilibrium $x_{0}=0$ and let $\tilde{x}(t)$ be double asymptotic solution to $x_{0} i . e .1 \mathrm{im} \tilde{x}(t)=x_{0}$ as $t \rightarrow \pm \infty^{\infty}$. In the extended phase space $(x, t)$, we have two asymptotic surfaces $W_{u}$, Wound by solutions tending asymptotically to $x_{0}$ as $t \rightarrow \underline{u}_{\infty}$ and $t++\infty$, respectively. In the unperturbed system they are doubled (coincide). For $E$ small enough, there exists hyperbolic $2 \pi-p e r i o d i c$ solution $x_{\epsilon}(t)$. In general, its asymptotic surfaces $\mathbb{F}_{u}^{\epsilon}$, $W_{s}^{\epsilon}$ do not coincide and cross transversely.

Points belonging to both of the surfaces are called homoclinic. Condition for transversal crossing of the separatrices can be expressed in terms of Melnikov's integral. Namely, if function:

$$
M\left(t_{0}\right)=\int_{-\infty}^{\infty}\left\{H_{0}, H_{1}\right\}\left(\tilde{x}\left(t-t_{0}\right), t\right) d t
$$

has simple zero then perturbed asymptotic surfaces cross transversely and hamiltonian system (1) is nonitegrable. In such situation, for the Poincaré's map $p_{\epsilon}$ of perturbed system following statements are valid:

1. $P_{\epsilon}$ has invariant structurally stable Cantor set $\Lambda$. 2. For some $N<\infty, \mathbf{P}_{\epsilon}^{N}$ on invariant set $\Lambda$ is topologica11 y conjugate to a $\epsilon$ shift on two symbols.

Invariant set $\Lambda$ contains:

1. A countable infinity of periodic orbits (including orbits with arbitrarily long period).

2. An uncountable infinity of nonperiodic orbits.

3. A dense orbit.

The explanation why, and in what sense, dynamics on $\Lambda$ can be interpreted as chaotic, one can find in [7].

More detailed study of some problems presented in this paper one can find in [10]. 


\section{PERTURBED PLANAR OSCILATION OF A RIGID SATELL ITE}

In this section we give few examples of nonitegrability of perturbed planar oscilation of a satellite in a circular or bit.

2.1 Perturbation due to eccentricity of the orbit

Let us consider a rigid body in an elliptic orbit (see

Fig. 1). Equation of its planar oscilation has the form [1]:

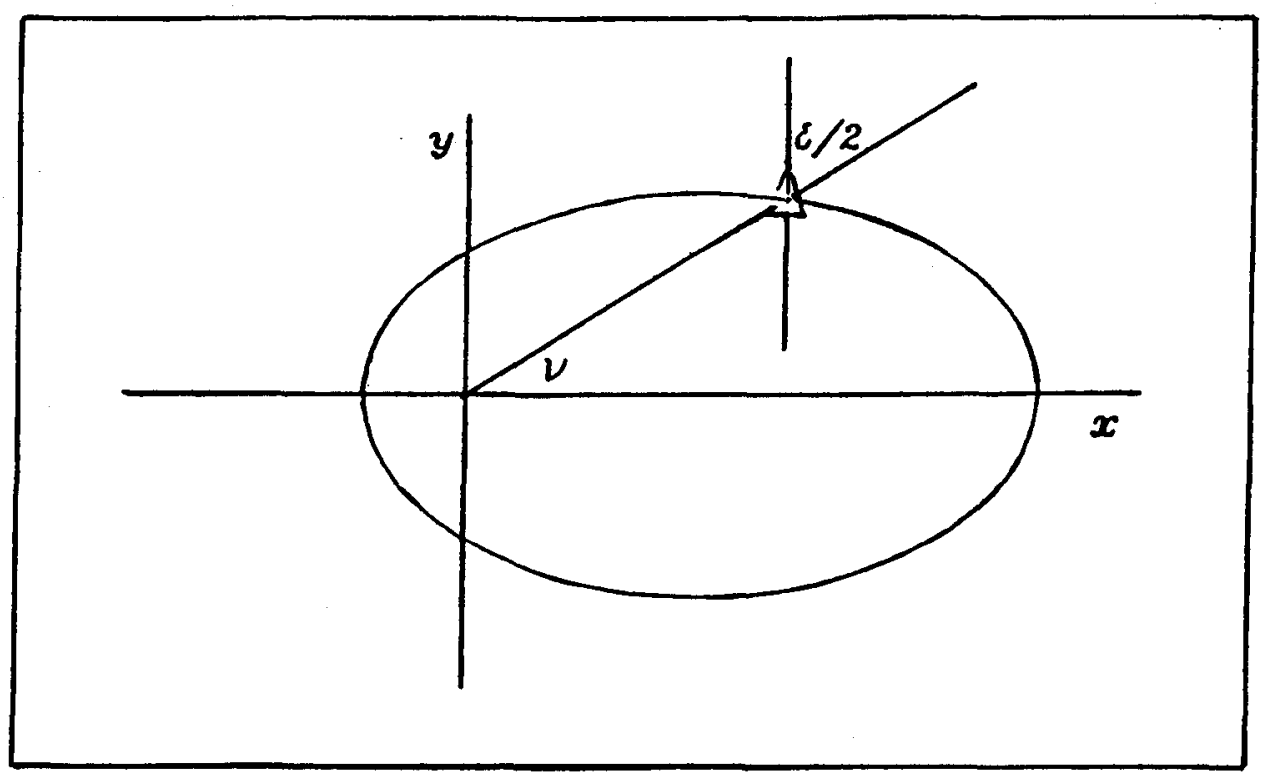

Fig. 1: Planar oscillation of a satellite in elliptic orbit.

$$
(1+e \cos (\nu)) \frac{d^{2} \delta}{d \nu^{2}}-2 e \sin (v) \frac{d \delta}{d \nu}+n^{2} \sin (\delta)=4 e \sin (v)
$$

where $e$ is the eccentricity, $\nu$ is the true anomaly, and $n>0$ is the parameter characterizing mass distribution of the body. This equation can be expressed in the Hamil tonian form: 


$$
\begin{gathered}
\frac{d q}{d \nu}=\frac{\partial H}{\partial p}, \frac{d p}{d}=-\frac{\partial H}{\partial q}, \\
H=\frac{1}{2}\left[\frac{p}{1+e \cos (\nu)}-2(1+e \cos (\nu))\right]^{2}-(1+e \cos (\nu)) n^{2} \cos (q)
\end{gathered}
$$

Eccentricity will be considered as small parameter. Hamiltonian function can be represented in the form (1) where:

$$
H_{0}=\frac{1}{2}(p-2)^{2}-n^{2} \cos (q), H_{1}=-\left[\left(p^{2}-4\right)+n^{2} \cos (q)\right] \cos (v)
$$

Unperturbed hyperbolic equilibrium solution (e $=0$ ) is given by:

$$
q(\nu) \equiv \pi, p(\nu) \equiv 2 .
$$

One can easily find two double asymptotic solutions to this equil ibrium:

$$
\begin{gathered}
p^{ \pm}(v)=2 \pm \frac{2 n}{\operatorname{ch}(n v)}, \\
\sin \left(q^{ \pm}(v)\right)= \pm \frac{\operatorname{sh}(n v)}{\operatorname{ch}^{2}(n v)}, \cos \left(q^{ \pm}(v)\right)=\frac{2}{\operatorname{ch}^{2}(n v)}-1
\end{gathered}
$$

Using method of residuals it is possible to calculate Melnikov's integral for both homoclinic loops:

$$
\begin{aligned}
M^{ \pm}\left(\nu_{0}\right) & =\int_{-\infty}^{\infty}\left\{H_{0}, H_{1}\right\}\left(q^{ \pm}\left(\nu-v_{0}\right), p^{ \pm}\left(v-v_{0}\right), v\right) d \nu \\
& =\frac{4 \pi}{\operatorname{sh}\left(\frac{\pi}{2 n}\right)}\left[ \pm t h\left(\frac{\pi}{2 n}\right)+\frac{3}{4}\right] \sin \left(\nu_{0}\right) .
\end{aligned}
$$

Thus, it is easy to see, that one pair of asymptotic surfaces (with sign plus) crosses transversely for any value of parameter $n>0$ and the system is nonitegrable. The second pair of asymptotic surfaces also crosses transver sely when $n \neq \pi / 1 n 7$. If $n=\pi / \ln 7$ then splitting of the separatrices has order smaller than e. Calculations presented above repeat those of Burov [4],[5] (we corrected some misprints). It should be al so noted that equation equivalent to (3) was used by Wisdom and 
co-workers [18] as the model of spin-orbit coupling for Hyperion. Numerical study of this equation allowed them to show chaotic rotation of Hyperion - known as the most evidend example of chaotic motion in the solar system.

\subsection{Solar radiation perturbations}

Let us consider rigid satellite in a circular orbit that lies in the ecliptic plane (see Fig. 2). We assume that torque caused by radiation pressure is perpendicular to the orbital plane (center of resultant radiation force lies on $x^{\prime}$ axis). Ve neglect shadowing effect. Fquation of planar oscillations of the satellite has the form:

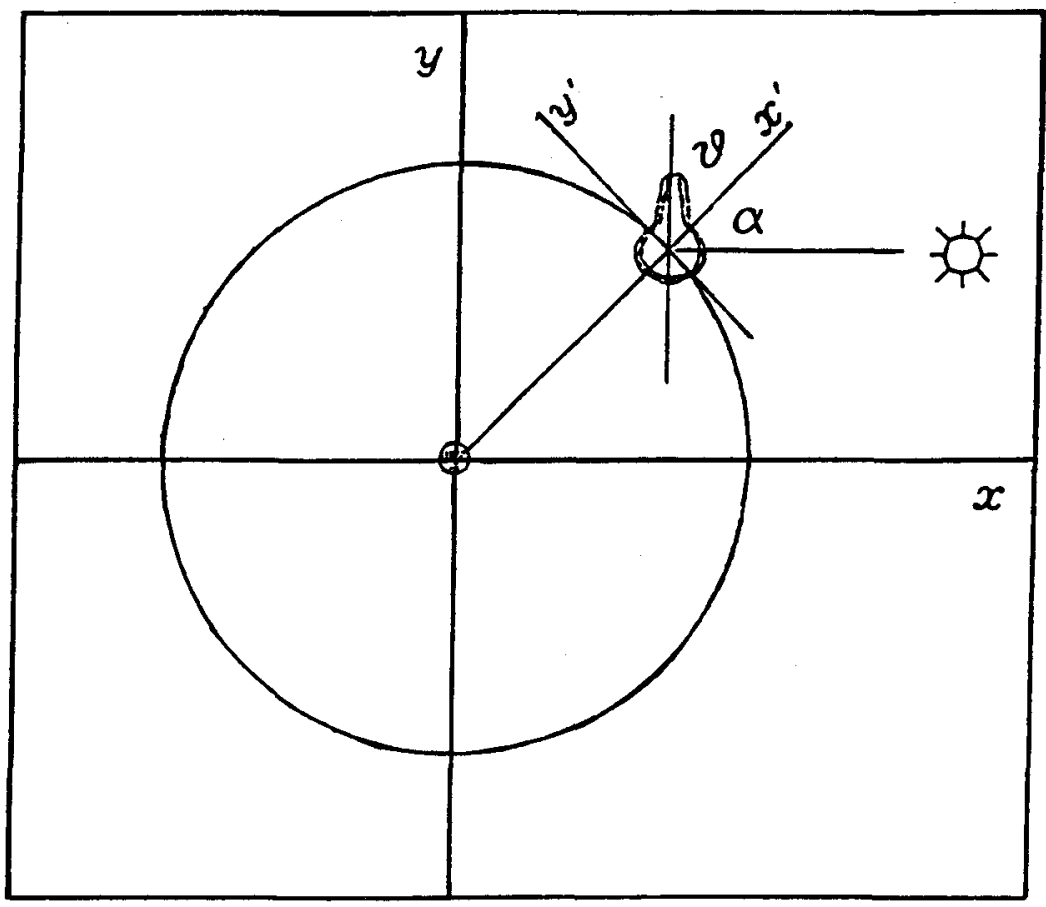

ig. 2: Perturbed planar oscillation of a satelite in circular orbit. Solar radiation pressure perturbations. 


$$
\frac{d^{2} \theta}{d \alpha^{2}}+\frac{1}{2} n^{2} \sin (2 \theta)+\frac{1}{2} \epsilon \sin (\theta+\alpha)=0 .
$$

This equation is equivalent to the Familton's equations:

$$
\frac{d q}{d \alpha}=\frac{\partial H}{\partial p}, \frac{d p}{d \alpha}=-\frac{\partial H}{\partial q}
$$

where Hamiltonian function is given by:

$$
H=\frac{p^{2}}{2}-n^{2} \cos (q)-\epsilon \cos \left(\frac{q}{2}+\alpha\right) \text {. }
$$

Snall parameter $\epsilon$ is proportional to the solar radiation torque, parameter $n$ depends on mass distribution of the satellite and $q=\theta / 2$. Unpertur bed equations $(4)(\epsilon=0)$ have hyperbolic equilibrium solution:

$$
\mathbf{q}(\alpha) \equiv \pi, \mathbf{p}(\alpha) \equiv 0
$$

and there exist two double asymptotic solutions:

$$
\begin{gathered}
\mathrm{p}^{ \pm}(\alpha)= \pm \frac{2 \mathrm{n}}{\mathrm{ch}(n \alpha)}, \\
\sin \left(\mathrm{q}^{ \pm}(\alpha)\right)= \pm \frac{2 \operatorname{sh}(n \alpha)}{\mathrm{ch}^{2}(n \alpha)}, \cos \left(\mathrm{q}^{ \pm}(\alpha)\right)=\frac{2}{\operatorname{ch}^{2}(n \alpha)}-1
\end{gathered}
$$

We can calculate Melnikov's integrals:

$$
\begin{aligned}
M^{ \pm}\left(\alpha_{0}\right) & =\int_{-\infty}^{\infty}\left\{H_{0}, H_{1}\right\}\left(q^{ \pm}\left(\alpha-\alpha_{0}\right), p^{ \pm}\left(\alpha-\alpha_{0}\right), \alpha\right) d \alpha \\
& =\frac{2 \pi}{n \operatorname{sh}\left(\frac{\pi}{2 n}\right)}\left[\operatorname{th}\left(\frac{\pi}{2 n}\right)-1\right] \sin \left(\alpha_{0}\right) .
\end{aligned}
$$

It is easy to observe that for any values of parameter $n$ the above function has simple zero. Thus, both pairs of a symptotic surfaces cross transversely and equations (4) are nonitegrable.

It should be noted that solar radiation torque depends strongly on satellite geometry. If the torque is caused by flat surfaces like big antennas or solar batteries then 
right hand sides of differential equations describing rotational motion of the satellite are only piecewise smooth. In these cases, for analysis of motion, special techniques should be used (see [14],[15]). Example of investigation of planar oscillation of an umbrella like satellite under influence of gravitational and solar radiation torques can be found [2].

\subsection{Third body perturbations}

Let us consider rotational motion of a rigid body in a circular orbit under influence of central body $M$ and 'moon' m whose orbit is assumed also circular and coplanar with orbit of the body (see Fig. 3 ). We approximate influence of 'moon' resolving potential of the torque with respect to the $r / R$ ratio, where $r$ is the radius of the body orbit and $R$ is radius of 'moon' orbit. For planar oscillations we obtained following equation:

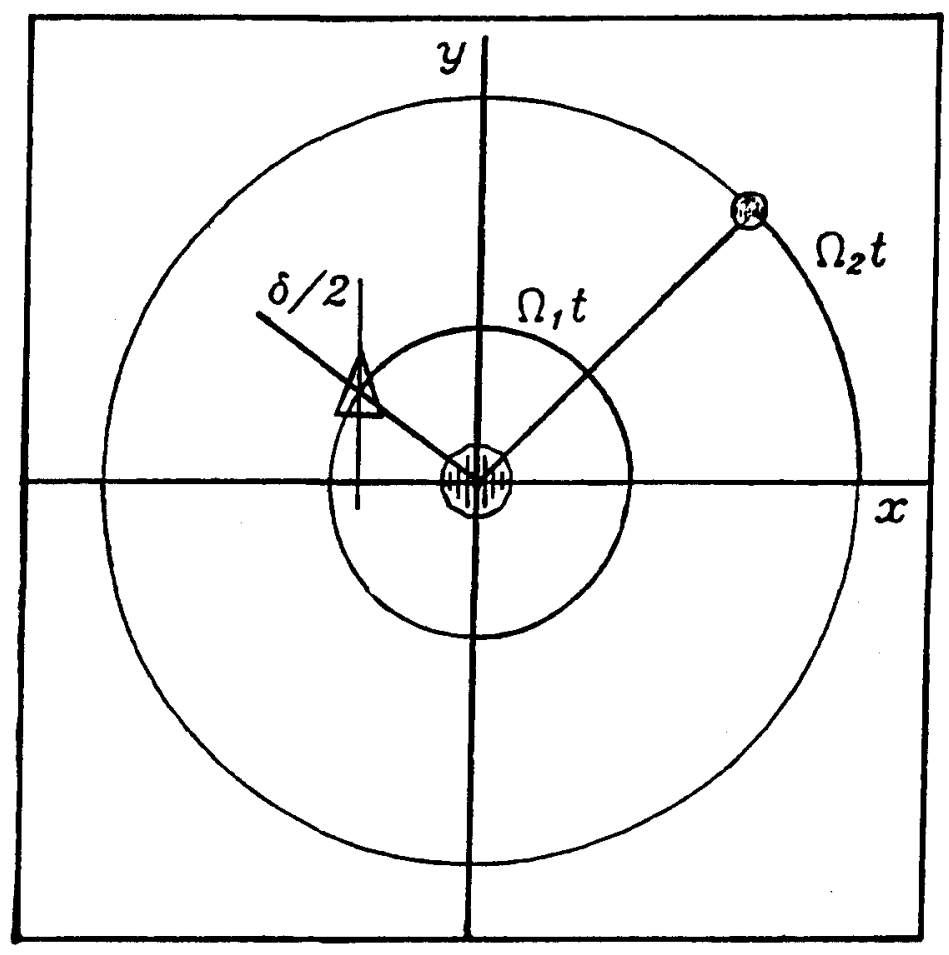

Fig.3: Perturbed planar oscillation of a satellite in circular orbit. Third body perturbations. 


$$
\frac{d^{2} \theta}{d \nu^{2}}+\frac{1}{2} n^{2} \sin (2 \theta)-\frac{1}{2} n^{2} \sin (\nu-2 \theta)=0
$$

where

$$
\begin{aligned}
v=\Omega t, \Omega=2\left(\Omega_{1}-\Omega_{2}\right), n^{2} & =3\left(\frac{\Omega_{1}}{\Omega_{2}}\right)^{2} \frac{\mathrm{B}-\mathrm{A}}{\mathrm{C}}, \\
\epsilon & \sim\left(\frac{\Omega_{2}}{\Omega_{1}}\right)^{2} \frac{\mathrm{m}}{\mathrm{M}},
\end{aligned}
$$

$\Omega_{1}$ and $\Omega_{2}$ are angular orbital velocities of the body and 'moon', respectively. As in previous cases we can write this equation in the form of Hamilton's equations with familtonian function:

$$
H=\frac{p^{2}}{2}-n^{2} \cos (q)-\epsilon n^{2} \cos (\nu-q)
$$

where

$$
q=2 \theta \text {. }
$$

Unperturbed system ( $\epsilon=0$ ) has hyperbolic equilibrium solution

$$
q(\nu) \equiv \pi, p(v) \equiv 0
$$

and two double asymptotic solutions:

$$
\begin{gathered}
p \pm(\nu)= \pm \frac{2 n}{\operatorname{ch}(n v)}, \\
\sin \left(q^{ \pm}(\nu)\right)= \pm \frac{\operatorname{sh}(n v)}{\operatorname{ch}^{2}(n v)}, \cos \left(q^{ \pm}(v)\right)=\frac{2}{\operatorname{ch}^{2}(n v)}-1
\end{gathered}
$$

Calculations show that Melnikov's integral in this case is:

$$
\begin{aligned}
M^{ \pm}\left(\nu_{0}\right) & =\int_{-\infty}^{\infty}\left\{H_{0}, H_{1}\right\}\left(q^{ \pm}\left(\nu-v_{0}\right), p^{ \pm}\left(v-v_{0}\right), v\right) d v \\
& =\frac{2 \pi n^{2}}{\operatorname{sh}\left(\frac{\pi}{2 n}\right)}\left[\left\{4\left(n^{2}+1\right) \pm n\right\} \operatorname{th}\left(\frac{\pi}{2 n}\right)-\frac{1}{n}\right] \sin \left(\nu_{0}\right) .
\end{aligned}
$$

This result shows that both pairs of asymptotic surfaces cross transversely and the system is nonitegrable. We note that the third body perturbations can be introduced in various 
ways. For example, one can consider the case when mass center of the body moves in infinitesimal periodic orbit around a libration point of the restricted $t$ hree body problem. Such a case for small periodic orbit around $L_{2}$ was analyzed in [6] where similar results were obtained.

\section{CHAOTIC ROTATIDNS OF SYMMETRIC RIGID BODY IN A CIRCULAR ORBIT}

In this section we consider a symmetric rigid body in a circular orbit. As the generalized coordinates we choose $\mathrm{Eu}-$ ler angles of the type 3-2-1. One coordinate is cyclic (because of the body symmetry). Using standard technique one obtains Hamiltonian function depending on two parameters:

$$
\begin{aligned}
H=\frac{1}{2}\left\{\frac{p_{1}+\gamma \sin \left(q_{2}\right)}{\cos \left(q_{2}\right)}\right\}^{2}+\frac{p_{2}^{2}}{2}-p_{1} & +\frac{3}{2}(\alpha-1) \cos ^{2}\left(q_{1}\right) \\
& \times \cos ^{2}\left(q_{2}\right)
\end{aligned}
$$

where

$$
\alpha=\frac{A}{B}, \gamma=\alpha \frac{\omega_{1}}{\omega_{0}},
$$

$\omega_{1}, w_{0}$ are the projection of absolute angular velocity of the body onto symmetry axis and orbital angular velocity, respectively. We will investigate the case when $\gamma=0$ and $\alpha=4 / 3$. There is special reason to investigate thoroughly this case. Sokolsky in his paper [16] studying stability of regular precessions (equilibria of vector field generated by (5)) made two remarkable hypotheses:

1. Cases $\alpha=1, \gamma=0$ and $\alpha=4 / 3, \gamma=0$ are transcendental.

2. The case $\gamma=0, \alpha=4 / 3$ is integrable.

First hypothesis states that stability of equilibrium can not be determined by coefficients of normal form of any finite order (for detailed discussion of this problem see [9]). The second one is based on the fact that the case $\alpha=1, \gamma=0$ is evidently integrable, so one can expect integrability in connection with transcendental cases of stability. We try to make some analytical approach in order to prove (non)integrability however, without success.

As an alternative we chose numerical exploration of the problem. For convenience we transformed the hamiltonian (5) to $t$ he form: 


$$
H=\frac{1}{2}\left\{\frac{2 p_{1}}{\cos \left(q_{2}\right)}\right\}^{2}+\frac{p_{2}^{2}}{2}-2 p_{1}+\frac{1}{4}\left(1+\cos \left(q_{1}\right)\right) \cos ^{2}\left(q_{2}\right)
$$

by means of canonical change of variables (now hamiltonian is $2 \pi$-periodic with respect to $q_{1}$ ). We restrict our analysis to the surface of constant energy $H=0$. This surface is compact. In the $\left(q_{1}, q_{2}, p_{2}\right)$ coordinates space equation $H=0$

defines bounded region, symmetric with respect to $\mathrm{p} 2=0 \mathrm{pla}$ ne. Figure 4 shows upper boundary of this region.

At first, we generated Poincare (global) cross section (see Fig. 5). As the plane of cross section we chose $q_{1}=\pi$. It is visible that on the plane of cross section there are two regions: chaotic in the central part of $f$ igure and ordered one in upper and lower parts (symmetric curves in upper and lower part of figure belong to one phase curve; all points in chaotic region belong to one phase curve). We found this feature of the cross section as untypical. Normally (e.g. Hennon-Heiles system) in the chaotic region there are 'islands' of ordered regions. In order to investigate this question we $f i x$ two windows ( $t$ wo squares in Fig. 5) one in the chaotic and second in the ordered region. Fig. 6 and Fig. 7 show that there are no chaotic parts. This and other tests made seem to support our statement about separation of the phase space into two regions. He also used Liapunov exponents to test if there is no ordered region in the chaotic part. Results are shown in Fig. 8, they also shows strict separation of the phase space.

Another interesting feature of the system can be observed if cross section plane is fixed on different places on $q_{1}$ axis (Figs. 9-11). When the plane of cross section is placed in lower values of $q_{1}$ axis then order region is smaller. For the case $q_{1}=\pi / 3$ it totally disappear. (In all figures where cross-section is shown position of plane of cross section is marked by $q_{1}^{c}$ ).

All these numerical observations seem to have some importance to the so called 'coexistence' problem formulated by $\mathrm{J}-\mathrm{M}$. Strelcyn [17]. It consist on proving frequently observed numerically chaotic and ordered behaviour of a dynamical system. In the system investigated above, all results seem to support hypothesis that we have here 'simple coexistence'. First explicit example of this kind of coexistence (for diffeomorfisms) was proved in [13]. 


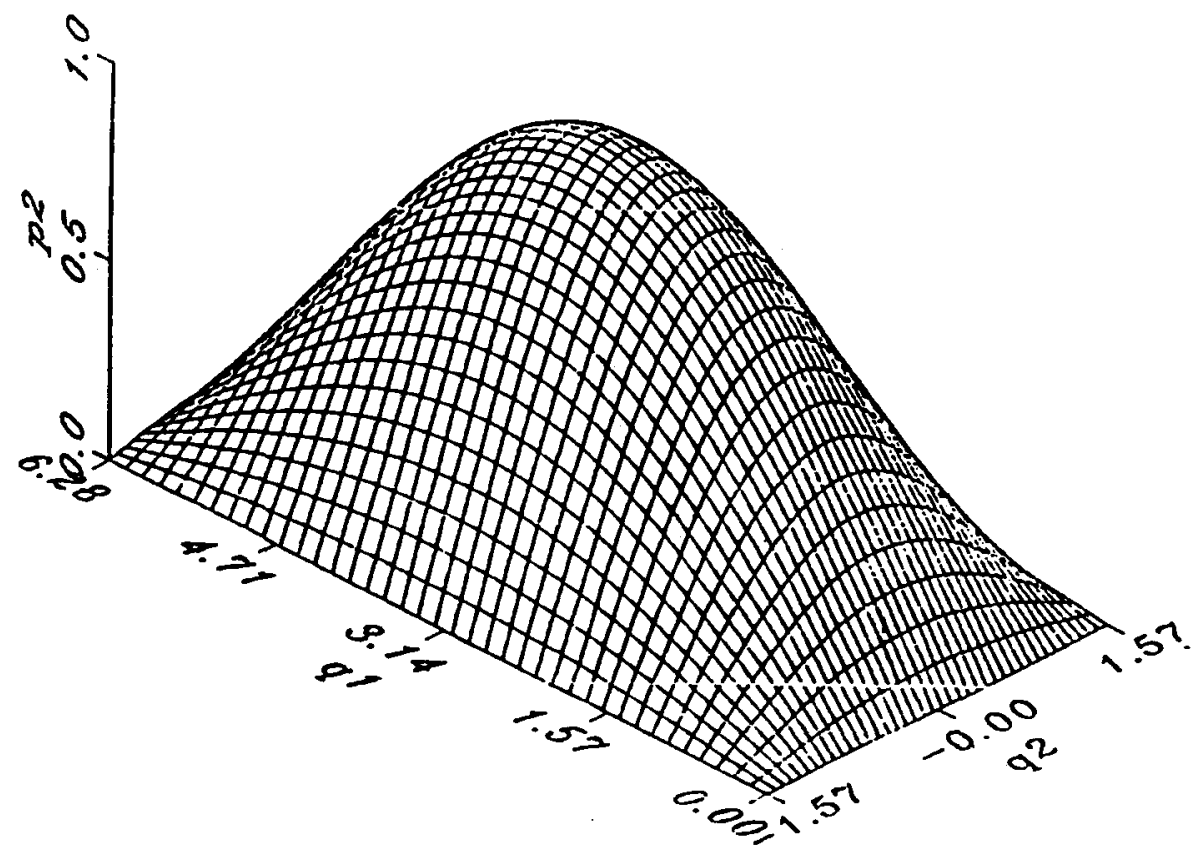

Fig. 4: Upper boundary of region $\mathrm{H}=0$ in the $\left(q_{1}, q_{2}, \mathrm{p}_{2}\right)$ coordinate space.

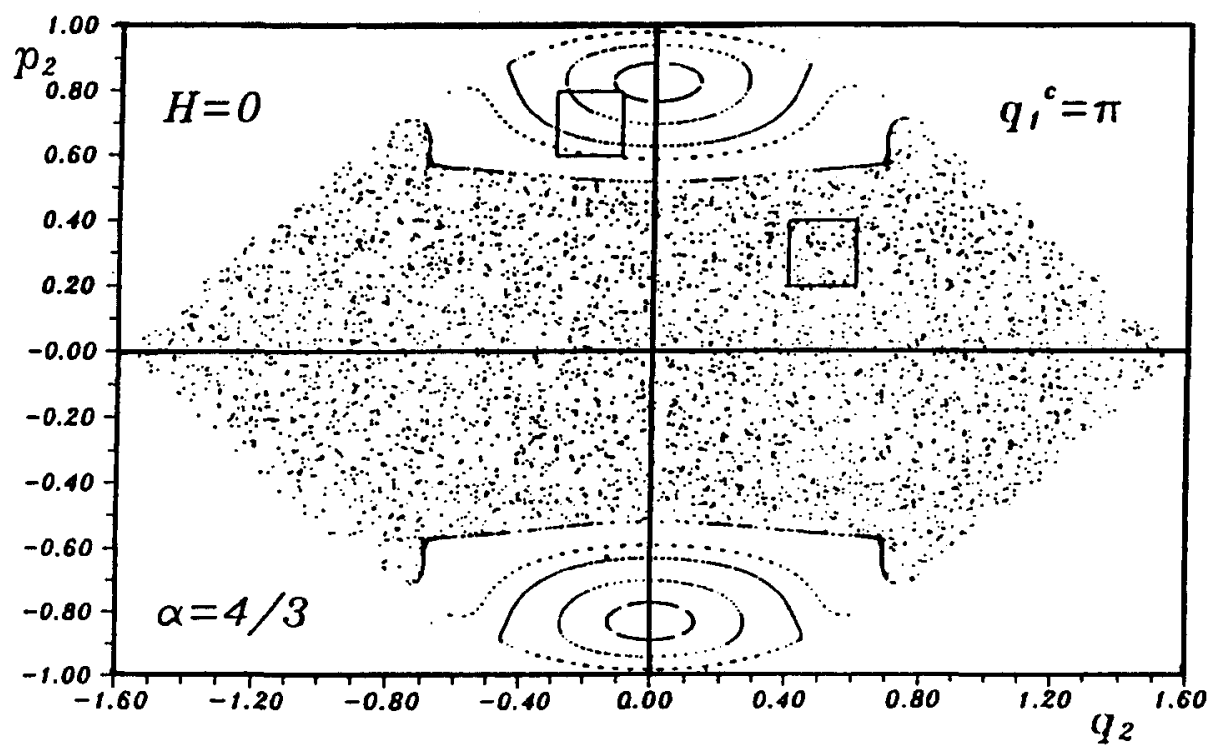

Fig. 5: Poincaré cross section. Two squares denote windows for more precise investigations. 


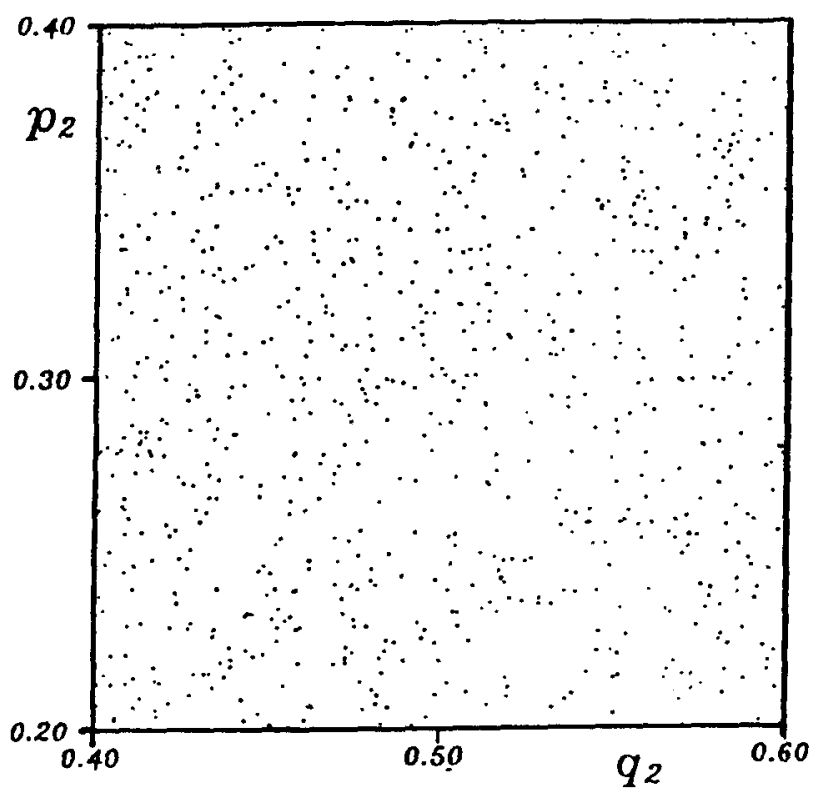

Fig. 6: Enlargement of chaotic window. Points belong to orbit different than this on Fig. 5.

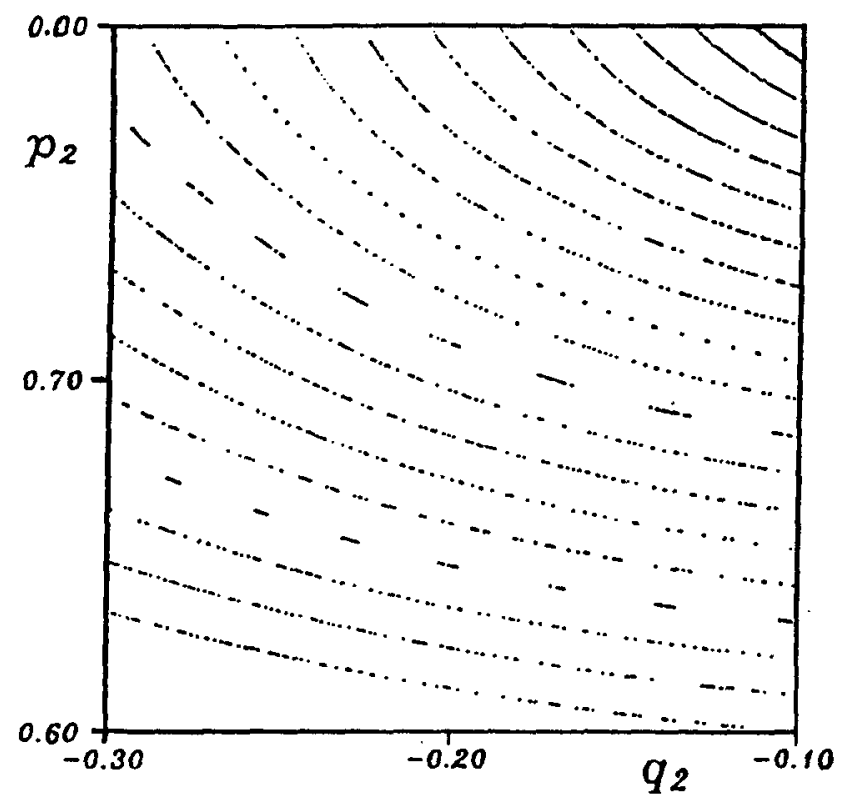

Fig. 7: Enlargement of ordered window. 


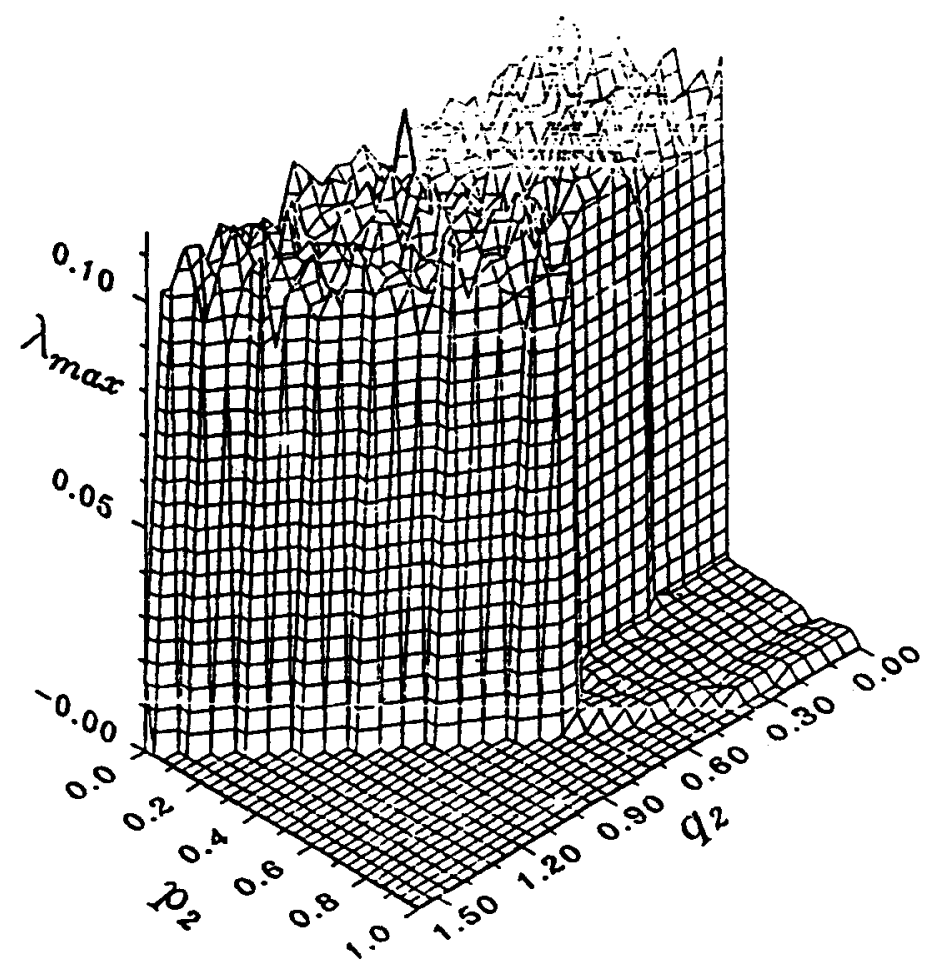

Fig. 8: Maximal Liapunov exponent as function of point on the cross section plane. For generation $30 \times 30$ grid of points was used.

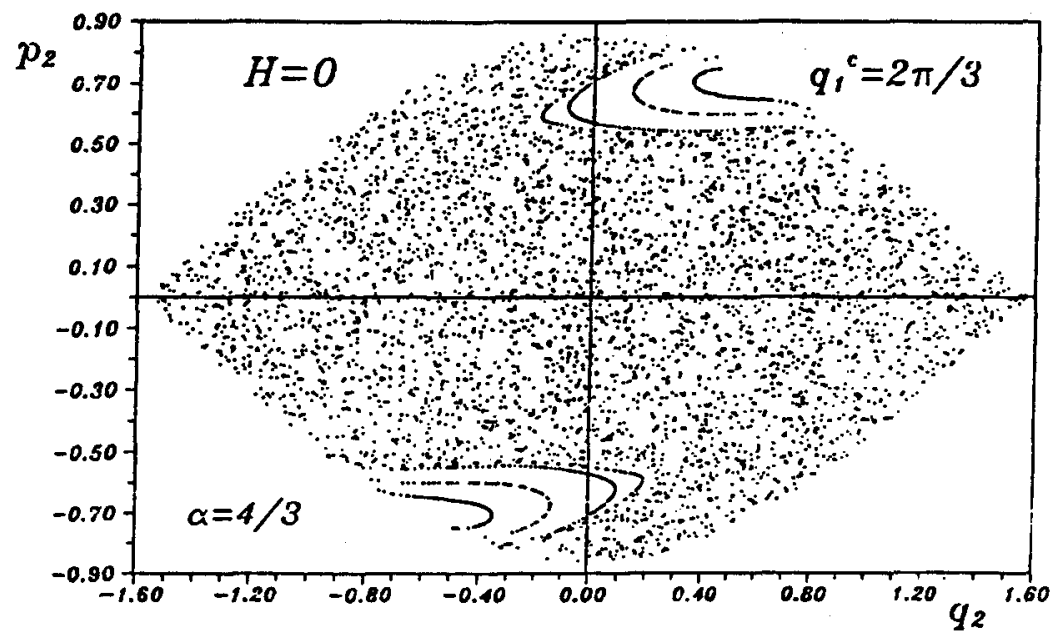

Fig. 9: Poincaré cross section. Plane of cross section located ar $\mathrm{q}_{1} \mathrm{c}=2 \pi / 3$. 


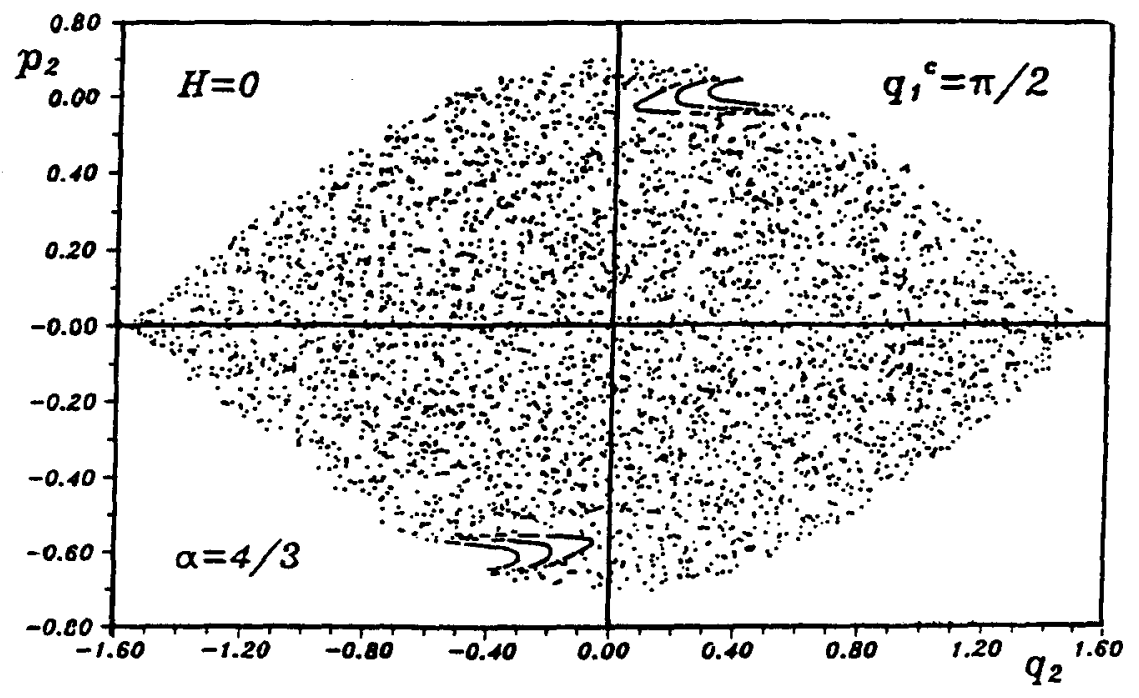

Fig. 10: Poincaré cross section. Plane of cross section located at $\mathrm{q}_{1}{ }^{\mathrm{c}}=\pi / 2$.

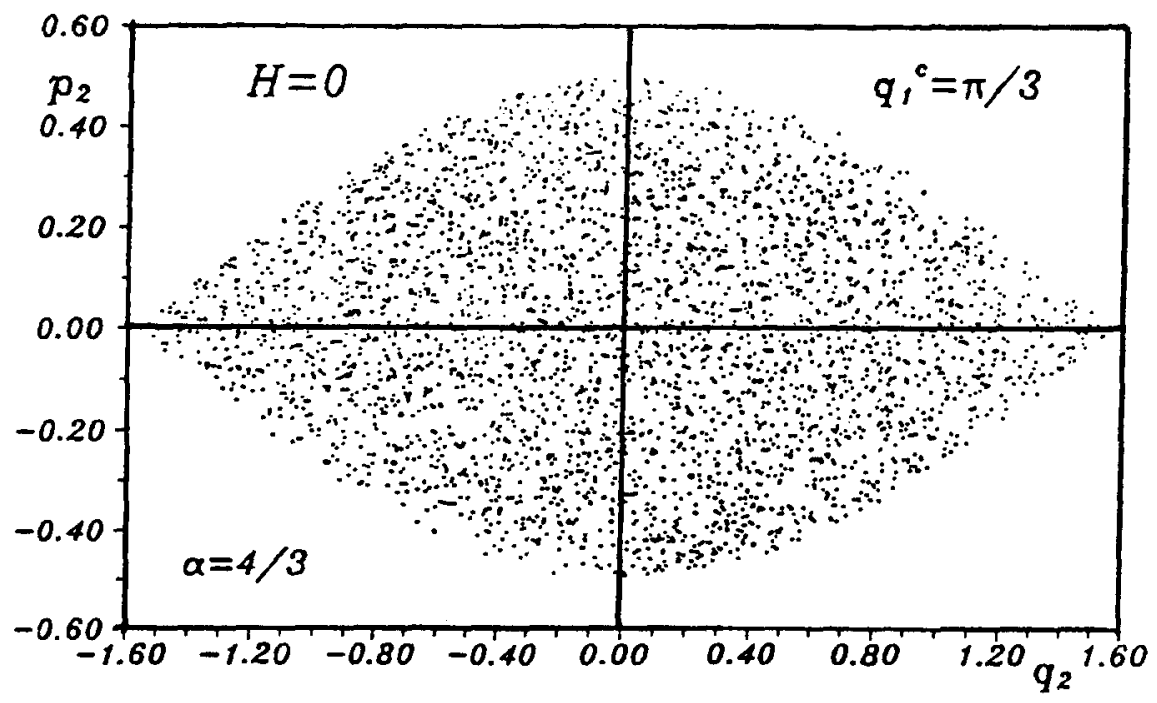

Fig. 11: Poincaré cross section. Plane of cross section located at $\mathrm{q}_{1} \mathrm{c}=\pi / 3$. 


\section{ACKNOWLEDGEMENTS}

I wish to express my appreciation to Prof. J-M.Streclyn for ours few days long discussion and advice and to K.Gozdziewski for his help in figures preparation. This work was supported by grant CPBR 12.20 and by UMK grant No. 253A.

\section{REFERENCES}

[1] Beletskii, V.V., 1965, 'The motion of an artificial satellite around a center of mass', Nauka, Moscow (in Russian).

[2] Beletski1, V.V., Starostin, E.L., 1990, 'Planar 1ibrations of a satelite under influence of gravity and solar radiation torques', Ko sm. Issled., 28, No.4,496-505.

[3] Bolotin, S.V., 1986, 'Liouville non-integrability conditions for Hamiltonian systems', Vestnik Moscow,Univ. Ser. Mat. Mech., No.3, 58-64.

[4] Burov, A.A., 1984, 'Nonitegrability of planar oscillations of a satellite in an elliptic orbit', Vestnik Moskov. Univ. Ser.Mat. Mech., No.1, 71-73.

[5] Aurov, A.A., 1984, 'About oscillations of a satellite in an elliptic orbit', Kossm. Issled., 22, No.1, 133-134.

[6] Churkina, N.I., 1988, 'The separatrices splitting and initiation of isolated periodic solutions in the problem of plane periodic motion of a satellite relatively to the mass center in a collinear libration point's vicinity', Ko sm. Issled., 26, No.3,472-474.

[7] Holmes, P., 1989, 'Nonl inear oscillations and the Smale horseshoe map', in Chaos and Fractals, (eds. R.L.Devaney, L. Keen), AMS, Vo1. 39.

[8] Kozlov, V.V., 1983, 'Integrability and non-integrability in Hamiltonian mechancs', Uspekhi Mat. Nauk, 38, No.1,367, (translation in Russian Math. Surveys, 1983,38, No.1, $1-76)$.

[9] Maciejewski, A.J., 1990, 'Transcendent cases of stability and hypotheses of Sokolsky', sukmitted to Celestial Mechanics.

[10] Maciejewski, A.J., 1990, 'Nonintegrability of perturbed planar oscillations of a satellite', in preparation.

[11] Melnikov, V.K., 1963, 'On the stability of the center for time periodic perturbations', Trudy Moskov. Mat.Obshch, 12,3-52 (translation in Trans.Moscow Math. Soc.,1983,12, $1-57$ ).

[12] Poincaré, H., 1972, 'Collected Works', Vol. I, II, Nauka, Moscow (in Russian).

[13] Przytycki, F., 1982, 'Examples of conservative diffeomorphisms of the two dimensional torus with coexistence of elliptic and stochastic behavior', Ergod.Th. and Dynam. Sys., 2,439-463. 
[14] Rybnikova, T.A., Bulatskaya, T.F., Rodnikova,A.V., 1988 , 'On stabilization of rotation of hel iocentric satellite with solar sale', Ko sm. Issled., 26, No.4, 625-628.

[15] Rybnikova, T.A., Treshchev, D.V., 1990, 'Existence of invariant tor $i$ in the problem of satellite with solar sale motion', Ko sm. Issled, 28, $\mathrm{Nb} .2,309-312$.

[16] Sokolsky, A.G., 1980, 'Problem of regular precessions stability of a symmetrical satellite', Kosm Issled.,17, No. 5, 698-706.

[17] Strelcyn, J.M., 1989, 'The "coexistence problem" for conservative dynamical systems a review', preprint No.89-13, Universite Paris Nord.

[18] Wisdom, J., Peale, S.J., Mignard, F., 1984, 'The chaotic rotation of Hyperion', Icarus, 58, 137-152.

[19] Ziglin, S.L., 1980,' Splitting of the separatrices, branching of solutions, and non-existence of an integral in the dynamics of a solid body', Trudy Moskov. Mat. Obshch., 41, 287-303 (translation in Trans.Moscow Matb. Soc., 1983, 41,283-298).

[20] Ziglin, S.L., 1987, 'Splitting of the separatrices and non-existence of an integral in a system of differential equations of Hamiltonian type with two degree of freedom', Izv. Akad. Nauk SSSR Ser.Mat., 51,1088-1103. 